\title{
ESTIMATING BLADDER CAPACITY IN CHILDREN
}

STEPHEN A. KOFF, M.D.

C.S. Mott Children's Hospital, and the Section of U rology,

University of Michigan Medical Center, Ann Arbor, Michigan

ABSTRACT - Bladder capacity in children can be estimated accurately by using the formula: Bladder capacity (ounces) $=$ age (years $)+2$.

The increased application of urodynamics to children has created a number of technical and interpretive problems not the least of which is a simplified age-adjusted standard for normalcy.

\section{Material and Methods}

In an attempt to formulate an estimate of ageadjusted normal values for bladder capacity, we studied 35 nonenuretic, uninfected children of different ages with cystometrics under anesthesia at the time of urologic surgery unrelated to the bladder (e.g., hypospadias repair). Actual bladder capacity was determined to be that volume above which the slope of the relatively flat cystometric curve abruptly changed and pressure per unit volume increased in a linear fashion. The results indicated that bladder capacity could be expressed as a simple formula related to the age of the child

Bladder capacity $($ ounces $)=$ age $($ years $)+2$

\section{Comment}

To validate this formula we compared it graphically to Starfield's* data which derived bladder capacity in 203 normal, nonenuretic children of different ages by measurements of voided volumes during diuresis. Figure 1 indicates that the two curves are nearly congruent.

While this formula is only an estimate of bladder capacity in children, which may be inaccurate

\footnotetext{
${ }^{*}$ Starfield, B.: Functional bladder capacity in enuretic and nonenuretic children, J Pediatr 70: 777 (1967).
}

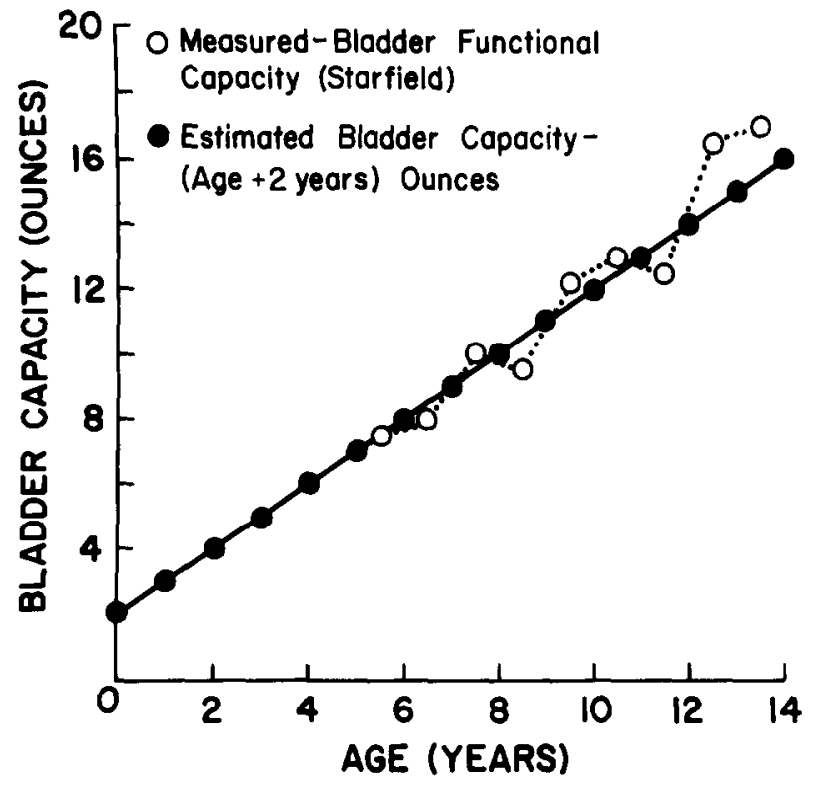

FIGURE 1. Graph of bladder capacity formula compared with Starfield's data.

at times, it has proved uscful in intcrpreting the pediatric cystometrogram to determine whether capacity is particularly large or small. It also has been helpful as a guide for calculating the maximal permissible urine volume in children on intermittent catheterization, and for determining whether or not the volume of contrast medium infused during voiding cystourethrography is physiologic.

Children's Hospital 700 Children's Drive Columbus, Ohiv 43205 